\title{
Efficient Hopfield pattern recognition on a scale-free neural network
}

Dietrich Stauffer ${ }^{1,2,4}$, Amnon Aharony ${ }^{1}$, Luciano da Fontoura Costa ${ }^{3}$ and Joan Adler 4

1 School of Physics and Astronomy, Raymond and Beverly Sackler Faculty of Exact Sciences, Tel Aviv University, Ramat Aviv, Tel Aviv 69978, Israel

2 Institute for Theoretical Physics, Cologne University, D-50923 Köln, Euroland

${ }^{3}$ Cybernetic Vision Research Group, IFSC-USP, Caixa Postal 369, 13560-970 São Carlos, SP, Brazil

4 Department of Physics, Technion-IIT, Haifa 32000, Israel

e-mail: aharony@post.tau.ac.il,stauffer@thp.uni-koeln.de,

luciano@if.sc.usp.br,phr76ja@techunix.technion.ac.il

Neural networks are supposed to recognise blurred images (or patterns) of $N$ pixels (bits) each. Application of the network to an initial blurred version of one of $P$ pre-assigned patterns should converge to the correct pattern. In the "standard" Hopfield model, the $N$ "neurons" are connected to each other via $N^{2}$ bonds which contain the information on the stored patterns. Thus computer time and memory in general grow with $N^{2}$. The Hebb rule assigns synaptic coupling strengths proportional to the overlap of the stored patterns at the two coupled neurons. Here we simulate the Hopfield model on the Barabási-Albert scale-free network, in which each newly added neuron is connected to only $m$ other neurons, and at the end the number of neurons with $q$ neighbours decays as $1 / q^{3}$. Although the quality of retrieval decreases for small $m$, we find good associative memory for $1 \ll m \ll N$. Hence, these networks gain a factor $N / m \gg 1$ in the computer memory and time.

PACS: 05.40-a, 05.50+q, 87.18.Sn

Traditional neural network models have nodes $i$ ("neurons") coupled to all other nodes $k$ with some coupling constant $J_{i k}$ ("synaptic strength"), similar to Sherrington-Kirkpatrick infinite-range spin glasses [1. Here we consider one of the simplest neural network models, due to Hopfield [2. This model was mostly applied to infinite range and was only rarely put onto a square lattice with short-range interactions [3, 4]. Real neural networks seem to have neither infinite nor only nearest-neighbour connections. The spatial structures of neural networks were investigated [5] and compared with small-world and scale-free networks [6, 7, 8, 9, 10, Now we present computer simulations of the Hopfield model [2] with Hebb couplings between neighbours restricted to a Barabási-Albert (BA) scale-free network [6].

In the Hopfield model, each of $N$ neurons or sites can be firing $\left(S_{i}=+1\right)$ or not firing $\left(S_{i}=-1\right)$. Neurons are coupled through $J_{i k}$, and are sequentially updated according to

$$
S_{i} \rightarrow \operatorname{sign}\left(\sum_{k} J_{i k} S_{k}\right)
$$


(We mostly ignore the diagonal terms $i=k$ in our sums.) This rule corresponds to a lowtemperature Monte Carlo simulation of a spin glass. The model has stored $P$ different patterns $\xi_{i}^{\mu}(\mu=1,2 \ldots, P)$, which we take as random strings of \pm 1 . The couplings are given by the Hebb rule:

$$
J_{i k}=\sum_{\mu} \xi_{i}^{\mu} \xi_{k}^{\mu} .
$$

The first of these patterns is presented to the network in a corrupted form $S_{i}$, with ten percent of the $S_{i}$ reversed in comparison to the correct $\xi_{i}^{1}$. The question is whether the iteration through Eq. (1) transforms the erroneous $S_{i}$ into the correct $\xi_{i}^{1}$. The quality of this pattern recognition is given by the overlap

$$
\Psi=\sum_{i} S_{i} \xi_{i}^{1} / N \quad,
$$

which is related to the Hamming distance and equals 1 for complete recognition and $\sim \pm 1 / \sqrt{N}$ for only accidental agreement at random sites; it is $\sim 0.8$ at the beginning of the pattern recognition process, due to the ten percent reversal.

Now we restrict the synaptic connections $J_{i k}$ to neurons which are neighbours in the BA network, but we still use Eqs. (1-3). In these networks, we start from a small core of $m$ sites, all connected with each other. Then $N \gg m$ nodes are added, one after the other. Each new site $i$ selects exactly $m$ sites from the already existing network sites as its neighbours $k$, with a probability proportional to the number of neighbours which the existing site $k$ has already: The rich get richer. When the network has added $N$ sites with a total of $N+m$ sites, its growth is stopped and the neural process of Eqs. (1-3) starts. Synaptic connections $J_{i k}$ exist only between sites $i$ and $k$ which are neighbours.

Since no longer every neuron is connected to all other neurons, the memory-saving trick of Penna and Oliveira [11] to avoid storing the $J_{i k}$ no longer applies. 400 Megabyte were needed for $N=10,000$ nodes and $P=20,000$ patterns. To save computer time, the $J_{i k}$ should be determined after and not before the construction of the BA network.

When only one pattern is stored, it is recognised completely after two iterations. With $P>1$, however, no complete recognition takes place, the overlap $\Psi$ is usually at the final fixed point (reached after about five iterations) lower than at the beginning, as shown in Fig. 1a . However, the model can still recognise the first pattern as the one presented to it, since the overlap $\Psi \sim 0.19$ for $P=N=10^{4}$ is still appreciably larger than the overlap $|\Psi|<500$ with the other $(P-1)$ patterns.

Rather similar results are obtained if we work on a nearest-neighbour hypercubic lattice with $N=L^{d}$ sites, similar to the studies made in [3, 4] in two dimensions. Fig.1b shows that only for small numbers $P$ of patterns an increased $d$ means an increased final overlap. For $d=7,10$ and 15 the overlaps with up to 20 patterns did not differ appreciably from $d=5$. No significant size effects were seen for $4 \leq L \leq 20$ in five and $4 \leq L \leq 13$ in seven dimensions. 
Fig. 1 is based on one sample only for each point; using instead 100 samples at $m=3$ and $N=10,000$, we see in Fig. 2 that the overlap varies roughly as $\Psi(P)-0.19 \propto P^{-0.6}$, except for very small $\mathrm{m}$. A similar power law $P^{-0.6}$ is also found for hypercubic lattices (not shown.) It would be interesting to understand this power law from some analytical analysis.

A much better recovery of the corrupted pattern is obtained if we take a larger inner core of the BA network, that means if $m$ is no longer small. (The first $m$ network sites are all mutually connected, as in the traditional Hopfield model.) Using 100 patterns, Fig. 3a shows the overlap $\Psi$ for $N+m=10^{4}$ total sites as a function of $m$. Already at $m=200, N=9800$ the final overlap is 88 percent; at $m=2000, N=8000$ we have complete recovery. For $1 \ll m \ll N$ the number of connections (counting each bond twice) is $m N$ in our case and $N^{2}$ in the fully connected case; thus we saved connections by a factor $m / N$. If we include the diagonal terms $J(i, i)=P$ in Eq. (1), we prevent the overlap from becoming worse than the initial overlap 0.8 for small $m$ and still get overlap near unity for large $m$, Fig. 3b.

Of course, with a large $m$ the network is no longer scale free, as shown in Fig. 4: The simple power law $\propto 1 / q^{3}$ for the number of sites with $q$ neighbours each [6] persists for $10^{2}<q<10^{3}$, but a Gaussian peak is added for large $q$. However, the additional bump concerns only a relatively small number of sites, and is probably negligible for any practical purposes.

For infinite range, $m=N$, the usual Hopfield model 12 gives an overlap $\Psi$ close to 1 for $P / N<0.14$ and a relatively small overlap $\Psi \sim 0.2$ for $P / N>0.14$, with a sharp jump at $P / N=0.14$. Our simulations, in contrast, show a gradual deterioration as soon as more than one pattern is stored, but the value of $\Psi$ is still of order 0.2 and distinctly larger than for the other $(P-1)$ patterns. Using a medium-sized fully connected core, like $m \sim 10^{3}$ at $P \sim 10^{2}$, surrounded by a larger BA network with $N \sim 10^{4}$ sites, gives a good compromise between good recovery and not too many connections.

So far it is not clear if the efficient recovery simply results from the relatively large average coordination number $m$, or by some additional ingredients in the problem. It would also be interesting if Nature takes advantage of a similar efficiency. If it does, do natural neural networks share some geometrical features with the large- $m$ (but finite) scale-free networks [6]?

We thank D. Horn for a discussion and the German-Israeli Foundation for supporting our collaboration.

\section{References}

[1] D. Sherrington and S. Kirkpatrick, Phys. Rev. Lett. 35, 1792 (1975).

[2] J.J. Hopfield, Proc. Natl. Acad. Sci. USA 79, 2554 (1982).

[3] B.M. Forrest, J. Physique 50, 2003 (1989). 
[4] K.E. Kürten, J. Physique 51, 1585 (1990).

[5] L. da F. Costa and E. T. M. Manoel, Neuroinformatics 1, 66 (2002); http://www.neuroinformaticsonline.com/

[6] A.L. Barabási and R. Albert, Science 286, 509 (1999); R. Albert and A.L. Barabási, Rev. Mod. Phys. 74, 47 (2002); S.N. Dorogovtsev and J.F.F. Mendes, Adv. Phys. 51, 1079 (2002).

[7] D.J. Watts and S.H. Strogatz, Nature 393, 440 (1998).

[8] O. Shefi et al., Phys, Rev. E 66, 021905 (2002).

[9] S. Morita et al., Physica A 298, 553 (2001).

[10] J. Karbowski, Phys. Rev. Lett. 86, 3674 (2001).

[11] T.J.P. Penna and P.M.C. de Oliveira, J. Phys. A 22, 2719 (1989).

[12] D. Volk, Int. J. Mod. Phys. C 9, 693 (1998) with earlier literature. 

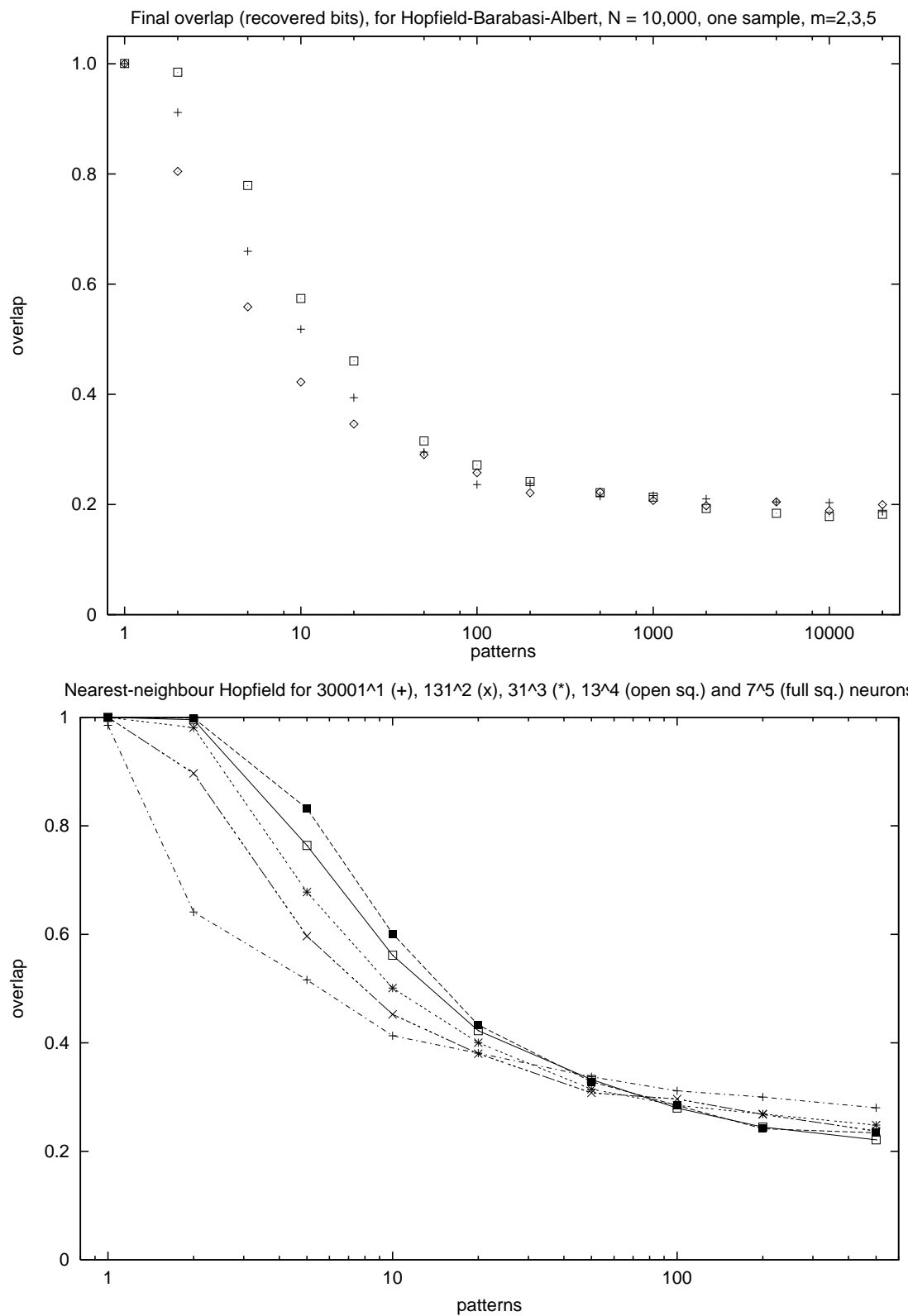

Figure 1: Final overlap $\Psi$ as a function of the number $P$ of patterns, for $N \sim 10^{4}$ neurons. Each point is based on one sample only. Part a: BA network with $m=2,3$ and 5 from bottom to top. Part b: Nearest-neighbour hypercubic lattice in one to five dimensions as shown in headline. 


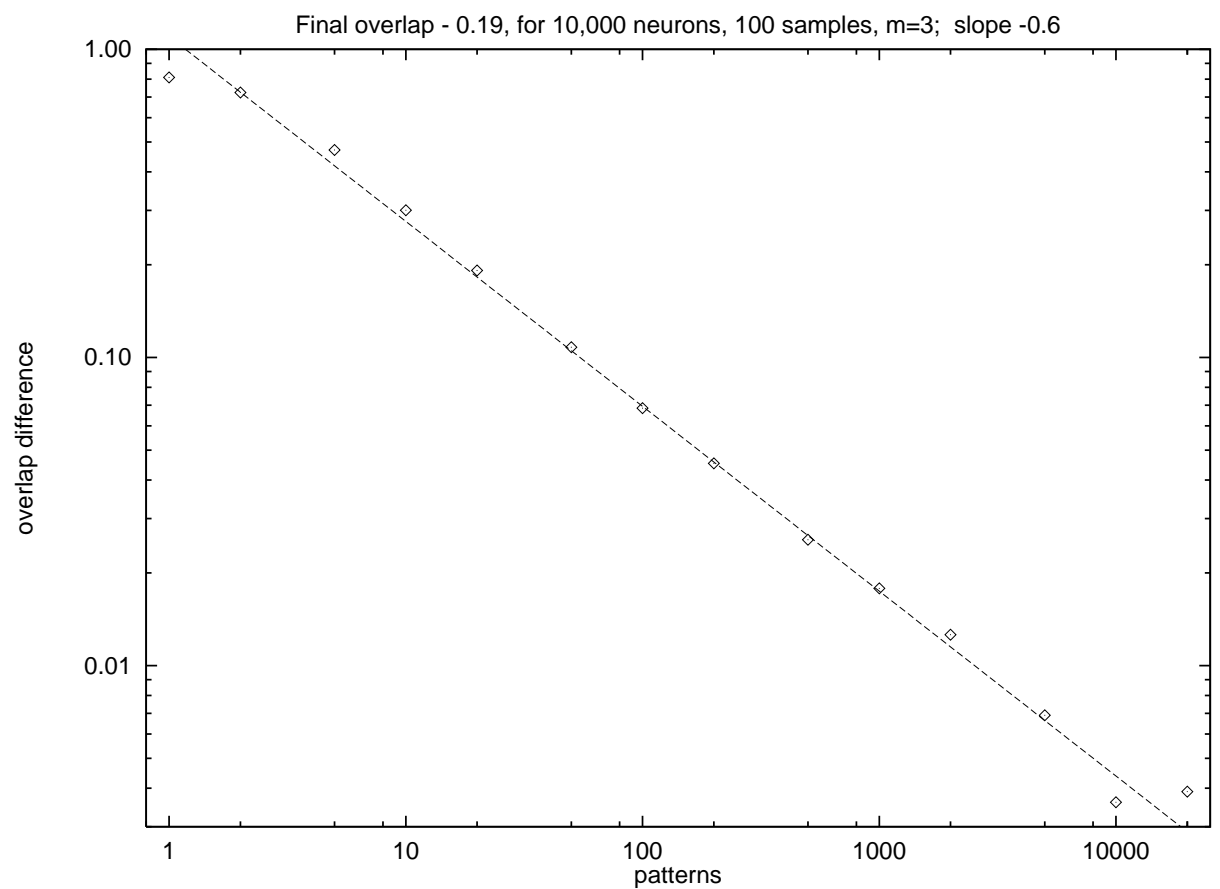

Figure 2: Approximate power law variation of final overlap difference $(\Psi-0.19)$ with the number of patterns $P$, averaged over 100 samples, with $N=10^{4}$ at $m=3$. The straight line has a slope -0.6 . 

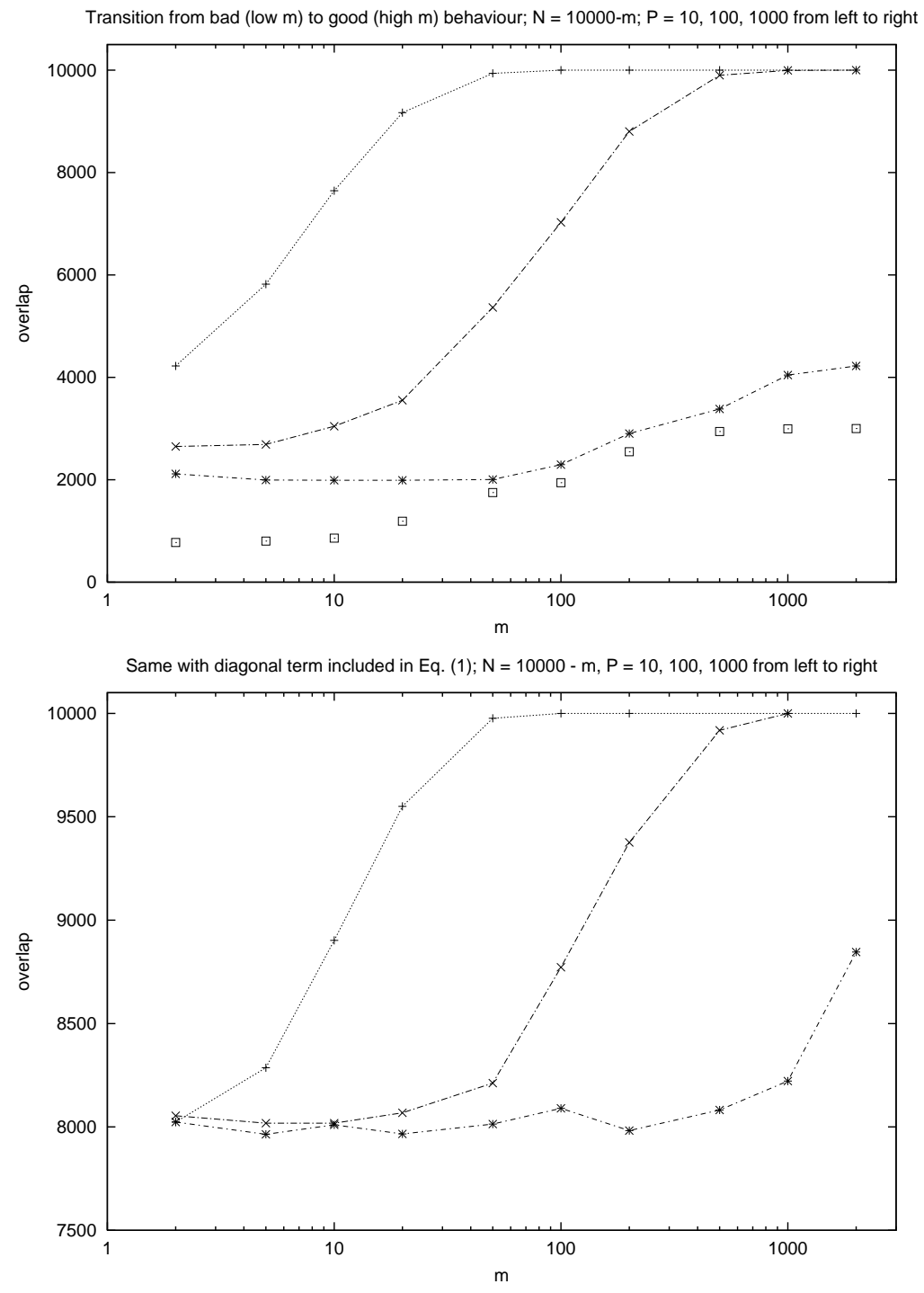

Figure 3: Variation of final overlap (not normalized) with the size $m$ of the fully connected core, surrounded by $N=10^{4}-m$ BA sites having $m$ neighbours each, at $P=10,100$ and 1000 (from left to right). Already for $P \ll m \ll N$ the corrupted pattern is restored well. The lowest data points refer to $P=100, N=3000-m$. Part a ignores the diagonal term in the sum (1), while part b includes it. 


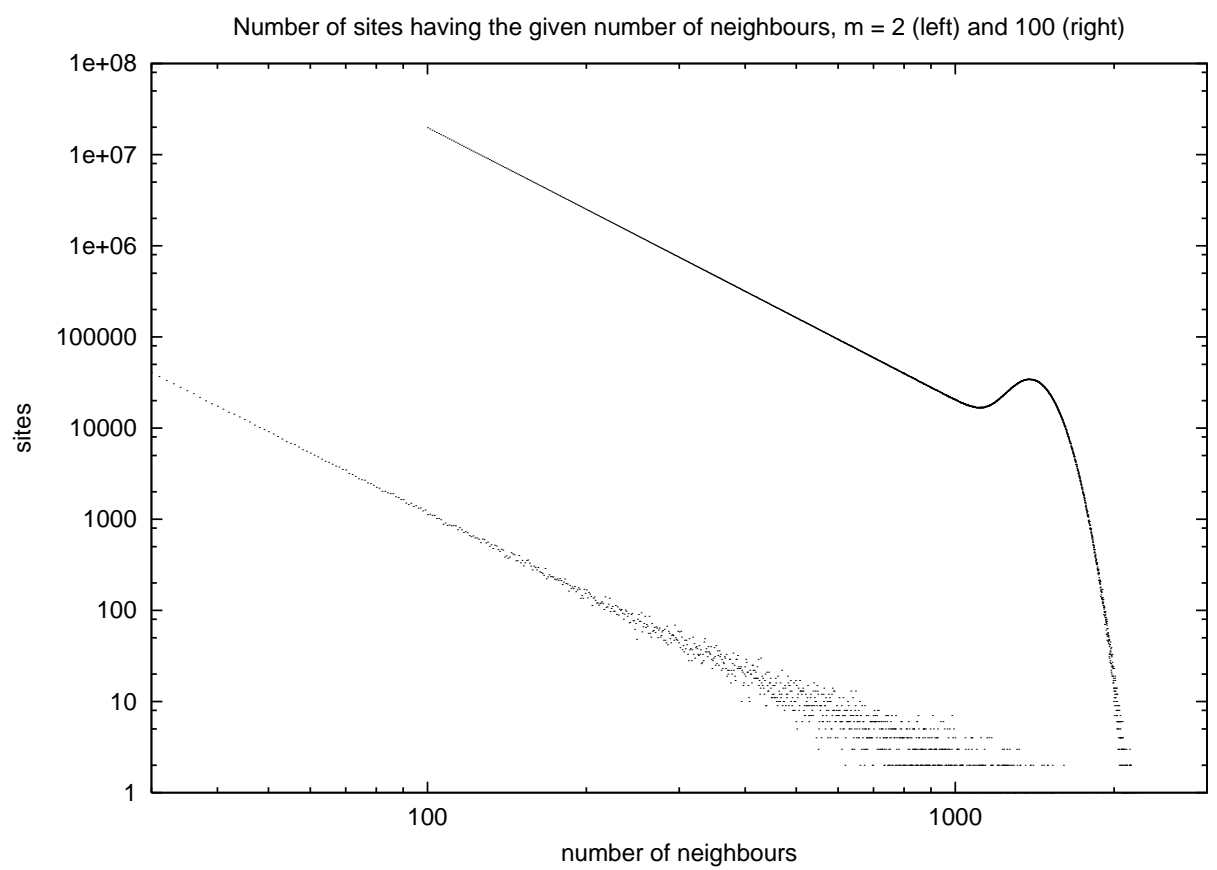

Figure 4: Number of sites having $q$ neighbours for $N=10^{4}$, summed over 100,000 simulations, at $m=100$ (right data). We no longer get the simple power law const $/ q^{3}$, shown here for comparison at $m=2$ (left data). 